\title{
Capacity analysis of threshold-based SNR scheduler in LTE systems
}

\author{
Ahmed lyanda Sulyman ${ }^{1 *}$, Ishtiaq Ahmad ${ }^{1}$, Hossam Hassanein ${ }^{3}$ and Saleh A Alshebeili, ${ }^{1,2}$
}

\begin{abstract}
This paper presents the capacity analysis of a threshold-based SNR scheduler in the long-term evolution (LTE) cellular systems. LTE standard has adopted multiuser OFDMA, and stipulates adjacent subcarrier groupings for mapping the physical OFDM subcarriers into resource blocks that form the basic unit of radio resource management (RRM) in LTE network. The standard however did not specify the details of the RRM algorithm to be employed, leaving this aspect for vendors to differentiate their products. Popular RRM algorithms such as roundrobin (RR), proportional fairness (PF), and maximum SNR (MaxSNR), have been implemented recently as operatorselectable options on LTE base station (BS). In this paper, we present a threshold-based SNR scheduler that has the capability of modeling all of the above-mentioned algorithms and thus allows vendors to combine the separate implementations of these algorithms into one generalized scheduling algorithm, where the threshold level used at any time instant defines the scheduling discipline to be realized. We derive the capacity enhancement achievable using the proposed scheduling scheme, and also present system-level simulations to corroborate the analysis. Our analytical and simulation results indicate that the proposed algorithm models the existing ones closely at different values of the threshold. The results also demonstrate the data rate enhancements, and the level of user fairness, achievable in the network for various levels of the threshold.
\end{abstract}

Keywords: Capacity analysis, Threshold-based scheduling, Broadband OFDMA, LTE networks, WiMAX networks

\section{Introduction}

OFDMA scheduling in the long-term evolution (LTE) system has received significant attentions lately as researchers and developers seek more efficient ways to handle the time-frequency resources in the fourth-generation $(4 G)$ networks [1-4]. The OFDMA specifications in the LTE standard allow only the grouping of adjacent subcarriers into logical subchannels known as physical resource blocks (PRB). This is in contrast to the WiMAX system which allows both adjacent subcarrier groupings known as band-AMC, as well as other pseudo-random mixing of subcarriers from different portion of the OFDM spectrums.

One of the many advantages of using adjacent subcarrier grouping in LTE is that the statistics of the subchannels or PRB's in the LTE system can be

\footnotetext{
* Correspondence: asulyman@ksu.edu.sa

${ }^{1}$ Department of Electrical Engineering, King Saud University, Riyadh, Saudi Arabia

Full list of author information is available at the end of the article
}

characterized reasonably well, making system planning and performance predictions easier.

Recently in [1], the author presents a threshold-based multiuser scheduling scheme for the WiMAX OFDMA systems. Threshold-based selection method was first proposed by Sulyman and Kousa in [5] for the diversity combining problem in a single-user transmission system, and has been widely studied in the literature [6-13]. In the context of multiuser scheduling in WiMAX OFDMA network, the author in [1] recently examines the use of a threshold-based multiuser scheduling, where a BS scheduler uses the energy threshold criterion [5-13], to select the users to be scheduled for downlink transmission in WiMAX network at any time instant.

In this paper, we examine further the applicability of this threshold-based SNR scheduling strategy in LTE networks. Due to analytical difficulty, the work in [1] only defines a performance metric called the throughput gain, and analyzes that metric as a measure of the enhancements possible using the threshold-based SNR

\section{Springer}


scheduling scheme in WiMAX OFDMA. The capacity analysis (or data rate enhancement possible) using threshold-based SNR scheduler is thus not yet analyzed. In the current paper, we derive accurate analytical expressions for the capacity enhancements provided by the proposed threshold-based SNR scheduler in the context of LTE OFDMA. Since the developed analysis is new in the literature, we also complement the analysis with a system-level simulation using the LTE PHY Lab and the LTE MAC Lab software provided in [14]. Both the analysis and simulation results indicate significant data rate enhancements using the proposed scheduling scheme, as the threshold level is increased in the range $[0,1]$. The results also indicate that at the extreme ends of the threshold, values " 0 " and " 1 ", the proposed scheduling scheme models the RR and MaxSNR schedulers respectively, while at different values of the threshold in between this interval, different flavours of user fairness vs. capacity enhancements can be realized. Thus the proposed scheduling algorithm can be implemented at LTE BS as a generalized scheduling algorithm that could replace the existing separate implementations of $R R$, MaxSNR, and PF, with a single algorithm capable of modeling all these scheduling disciplines whenever needed. Several kinds of generalized schedulers were explored extensively for high speed packet data access (HSPDA) or 3G in general, but have not been as much explored for the LTE. Given that HSPDA scheduling involves only time-domain scheduling decisions, while LTE involves both time- and frequency- domain scheduling decisions via the use of the PRB's and the TTI's, there is a significant difference between works done for HSPDA and the one presented for LTE in this paper, emphasizing the contribution of this work.

\section{System model and analysis}

\section{System model for threshold-based SNR scheduling in LTE} systems

In the LTE OFDMA specification, PRB is the basic unit of resource allocation per user and one PRB consists of twelve OFDM subcarriers, taken from adjacent portions of the OFDM spectrum.

Consider a downlink scheduler at the BS in an LTE system employing threshold-based SNR scheduling algorithm as shown in Figure 1, where a total of $N_{\mathrm{c}}$ PRBs are available in each transmission time interval (TTI). On each time slot, the BS scheduler schedules certain active users for downlink transmission, out of total of $L$ users, whose SNRs in the available PRBs meet or exceed a predetermined SNR threshold, $\gamma_{t h}$. The data of these users then fill the frequency-time resources in the next slots $[15,16]$.

We notice from our extensive simulations using system-level LTE simulator [14], that the user channel statistics do not change too rapidly across adjacent PRBs. Therefore instead of a scheduling process for each $\mathrm{PRB}$ as it is done in the existing schedulers, we can conduct a scheduling operation that would roughly be valid for a few number of adjacent PRBs. The threshold-based scheduler relies on this principle. Thus in the thresholdbased SNR scheduling scheme, the multi-dimensional scheduling problem of selecting a user per PRBs from among the SNR sets: $\left\{\gamma_{1}, \gamma_{2}, \cdots, \gamma_{L}\right\}_{1},\left\{\gamma_{1}, \gamma_{2}, \cdots, \gamma_{L}\right\}_{2}, \ldots$, $\left\{\gamma_{1}, \gamma_{2}, \cdots, \gamma_{L}\right\}_{N c}$, is closely approximated by the simpler scheduling process whereby $n$ users whose SNR are above a chosen SNR threshold are selected at a particular decision point in frequency domain, and this decision is utilized across $n$ adjacent PRBs which we term here the threshold PRBs (T-PRB). Thus scheduling is only

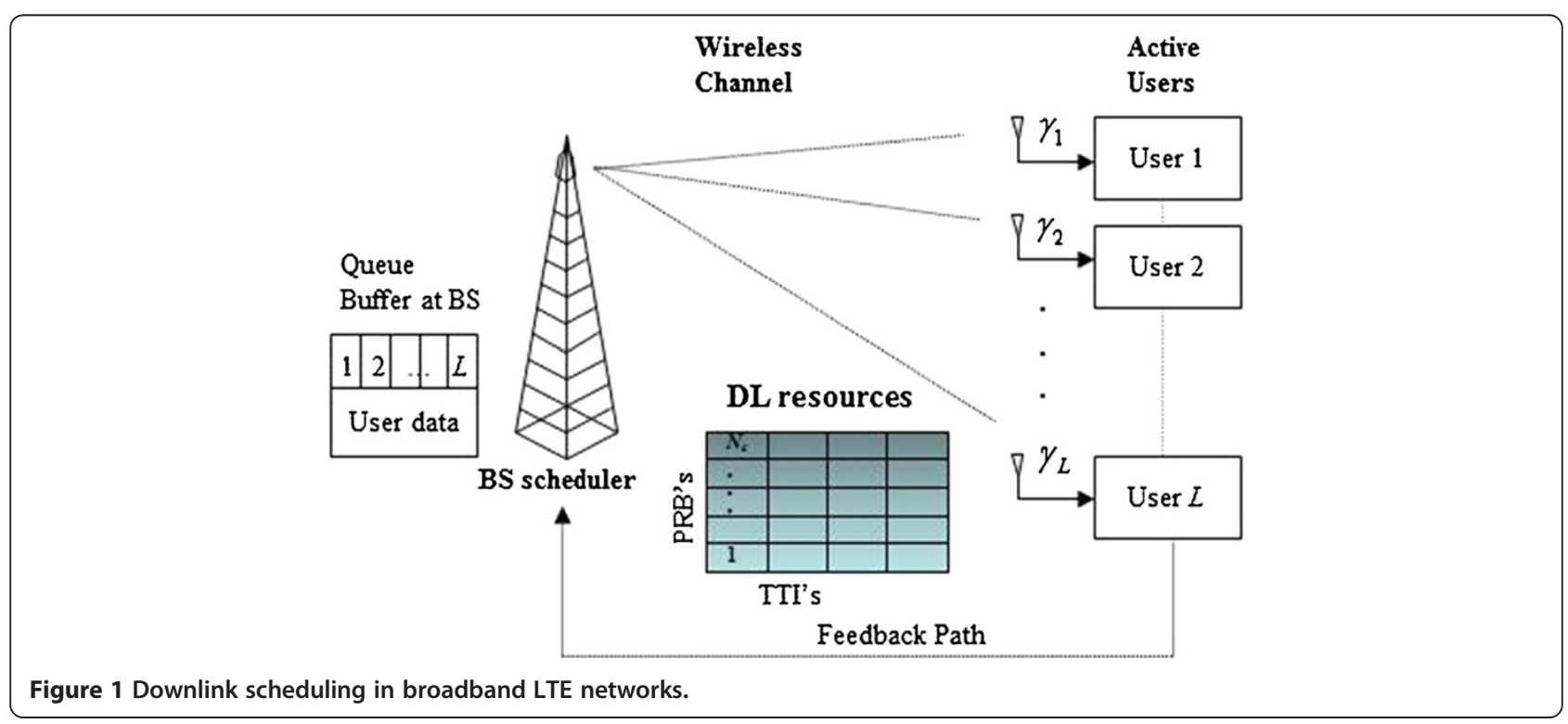


done at every $n$ PRB intervals in the frequency domain in the proposed scheme, resulting in a faster scheduling decision. As shown later on in our simulations, despite this procedural modification, the proposed scheduler still models the existing schedulers accurately at the appropriate values of the SNR threshold. The number of users, $n_{i}$, satisfying the threshold requirement on the $i^{\text {th }} \mathrm{T}$-PRB at any time instant, is not fixed but variable in correspondence to the user channel statistics on the available $N_{\mathrm{c}}$ PRBs, and the SNR threshold chosen. Notice that whenever $n_{i}=1$, a T-PRB becomes a PRB, and the scheduling decisions made in such cases are valid only for one PRB. The specific realization of $n_{i}$ could take any value from the set $\{1,2, \cdots, L\}$, at each scheduling decision point in frequency domain.

Let $\left\{\gamma_{1}, \gamma_{2}, \cdots, \gamma_{L}\right\}$ denote the set of SNRs of the $L$ users fed back to the BS on the $i^{\text {th }}$ T-PRB, and let $\left\{\gamma_{l: L}\right\}$ $l=1{ }^{L}$ denote the order statistics obtained by arranging these SNRs in decreasing order of magnitude, (i.e., $\gamma_{1: L} \geq$ $\left.\gamma_{2: L} \geq \cdots \geq \gamma_{n: L} \geq \gamma_{n+1: L} \geq \cdots \geq \gamma_{L: L}\right)$. As proposed in [5], define the SNR threshold as

$$
\gamma_{t h}=\mu \cdot \gamma_{1: L}
$$

where $0 \leq \mu \leq 1$. Then the BS scheduler conducts threshold test on the $i^{\text {th }}$ T-PRB and schedules the $n_{i}$ users whose SNR rank in the set $\gamma_{1: L} \geq \gamma_{2: L} \geq \cdots \geq \gamma_{n_{i}: L} \geq \gamma_{t h}$

Threshold-based SNR scheduling as explained above is a generalized scheduling scheme that can be used to model several other LTE scheduling schemes, depending on the threshold value $\mu$ chosen at any time instant. For example for $\mu=1$, the scheme reduces to MaxSNR scheduling, while the case $\mu=0$ corresponds to the RR scheduling policy where all users with strong and weak channels are given equal transmission opportunities. As $\mu$ is varied, in the range $0<\mu<1$, a whole range of user fairness vs. capacity enhancements can be realized. We also show that the well-known PF can be modeled in this range. Thus a threshold-based SNR scheduler when implemented on a BS would provide a simpler, yet efficient, generalized scheduling policy that would combine the separate implementations of the RR, MaxSNR, and PF algorithms, currently available in LTE BS [16], into one single algorithm where the threshold definition simply dictates the specific scheduling policy: RR, MaxSNR, $\mathrm{PF}$, etc., to be realized at any time instant, optimising cost and performance.

\section{Capacity analysis for threshold-based SNR scheduler in LTE systems}

Broadband transmissions in 4G LTE networks are typically arranged in bursts, with the basic unit of time denoted as TTI [16]. Assuming consecutive transmissions of length $N_{T T I}$ TTI's in total. At any time instant, users feedback their SNR in each PRBs to the BS, using the assigned pilots in the different PRB's, and a thresholdbased SNR scheduler conducts threshold test to select the $n_{i}$ users whose SNRs, $\gamma_{1}, \gamma_{2}, \cdots, \gamma_{n_{i}}$, are above the chosen threshold in the $i^{\text {th }}$ T-PRB and schedules them for downlink transmissions on $n_{i}$ adjacent PRBs, in the next TTI.

Let $n=\max \left\{n_{1}, n_{2}, \cdots n_{m}\right\}$, where $m$ is the total number of T-PRBs (or block-fading PRBs) available in the system at any time instant. For analysis convenience, we assume that for the case $n_{i}<n$ for a given T-PRB, the BS fills the remaining time-frequency transmission resources opportunistically by allocating them to the user with the best SNR in the respective T-PRB's. Thus without loss of generality, we assume that $n_{1}=n_{2}=\cdots=n_{m}$ $=n$ in the analysis. The impact of this assumption is that the capacity enhancement estimated in the analysis is less than what would be obtained in practice using threshold-based SNR scheduler in LTE networks.

For DL transmissions in LTE OFDMA system, the normalized BS capacity for each TTI is given by:

$$
C_{B S, \operatorname{perTTI}}=\sum_{k=1}^{N_{c}} \log _{2}\left(1+\gamma_{k}\right)
$$

where $\gamma_{k}$ denotes the SNR of the user being serviced on the $k^{\text {th }}$ PRB at any time instant. Considering a burst of length $N_{T T I}$ TTI's, then we can express the average BS data rate using the threshold-based SNR scheduler as

$$
C_{B S}=E\left[\sum_{t=1}^{N_{T T I}} \sum_{k=1}^{N_{\mathrm{c}}} \log _{2}\left(1+\gamma_{k}\right)\right]
$$

If we assume iid user channel statistics, then the longrun BS average data rate over each TTI will roughly be the same. Thus we can estimate the BS average capacity enhancements as

$$
C_{B S} \approx N_{T T I} E\left[\sum_{k=1}^{N_{c}} \log _{2}\left(1+\gamma_{k}\right)\right]
$$

Let $\hat{r}=\sum_{k=1}^{N_{c}} \log _{2}\left(1+\gamma_{k}\right)$, then the average BS data rate per TTI in the threshold-based SNR scheduling scheme is given by

$$
\begin{aligned}
r & =E[\hat{r}] \\
& =E_{n}\left\{E_{\gamma 1: L}, \ldots, E_{\gamma n: L}\left[\left.m \sum_{l=1}^{n} \log _{2}\left(1+\gamma_{l: L}\right)\right|_{\mathrm{n}}\right]\right\}
\end{aligned}
$$

where $\left\{\gamma_{l: L}\right\}_{l=1}^{L}$ is the order statistics obtained by arranging the set of user SNRs $\left\{\gamma_{j}\right\}_{j=1}^{L}$ in decreasing order of 
magnitude, (i.e., $\gamma_{1: L} \geq \gamma_{2: L} \geq \cdots \geq \gamma_{n: L} \geq \gamma_{n+1: L} \geq \cdots \geq \gamma_{L: L}$ ), and the parameter $m$ is as defined above. Assuming that the set $\left\{\gamma_{j}\right\}_{j=1}^{L}$ are iid, then the joint probability distribution function ( $p d f), \quad f_{\gamma_{1: L}, \cdots, \gamma_{L: L}}\left(\gamma_{1: L}, \cdots, \gamma_{L: L}\right)$, of $\left\{\gamma_{l: L}\right\}_{l=1}^{L}$ is given by [17]

$$
\begin{aligned}
f_{\gamma_{1: L}, \cdots, \gamma_{L: L}}\left(\gamma_{1: L}, \cdots, \gamma_{L: L}\right) & =L ! \prod_{i=1}^{L} f_{\gamma}\left(\gamma_{i: L}\right), \infty \\
& >\gamma_{1: L} \geq \gamma_{2: L} \geq \cdots \geq \gamma_{L: L}>0
\end{aligned}
$$

where $f_{\gamma}(\gamma)$ denotes the $p d f$ of the random variables $\gamma$.

Consider the subset $\left\{\gamma_{l: L}\right\}_{l=1}^{n}$ designating the $n$ largest $\gamma_{j}$ 's (corresponding to the $n$ users with the best SNRs scheduled for downlink transmission per spectrum access, $n \leq L)$. Then the joint $p d f, f_{\gamma_{1: L}, \cdots, \gamma_{n: L}}\left(\gamma_{1: L}, \cdots, \gamma_{n: L}\right)$, of $\left\{\gamma_{l: L}\right\}_{l=1}^{n}, n \leq L$, can be obtained as $[17,18]$

$$
\begin{aligned}
& f_{\gamma_{1: L}, \gamma_{2: L}, \cdots, \gamma_{n: L}}\left(\gamma_{1: L}, \gamma_{2: L}, \cdots, \gamma_{n: L}\right) \\
& =\int_{0}^{\gamma_{m: L}} \cdots \int_{\gamma_{n+2: L}}^{\gamma_{n: L}} f_{\gamma_{1: L}, \gamma_{2 L}, \cdots, \gamma_{L: L}}\left(\gamma_{1: L}, \gamma_{2: L} \cdots, \gamma_{L: L}\right) \quad d \gamma_{n+1: L}, \cdots, d \gamma_{L: L}, \\
& =n !\left(\begin{array}{l}
L \\
n
\end{array}\right)\left[F_{\gamma}\left(\gamma_{n: L}\right)\right]^{L-n} \prod_{l=1}^{n} f_{\gamma}\left(\gamma_{l: L}\right), \gamma_{1: L} \geq \gamma_{2: L} \geq \cdots \geq \gamma_{n: L} .
\end{aligned}
$$

(6) where

where $\left(\begin{array}{l}L \\ n\end{array}\right)=(L ! / n !(L-n) !)$ denotes the binomial coefficient, and $F_{\gamma}(\gamma)=\int_{0}^{\gamma} f_{\gamma}(\gamma) d \gamma$ is the cumulative distribution function $(c d f)$ of the random variables $\gamma_{j}^{\prime}$ s. We consider that the underlying user channels experience
Rayleigh fading, therefore the $\left\{y_{i}\right\}_{j=1}{ }^{L}$ are exponentially distributed, with pdf, $f_{X}(x)$, and cdf, $F_{\gamma}(\gamma)$, given by [17]

$$
\begin{aligned}
& f_{\gamma}(\gamma)=a \exp (-a \gamma), \\
& F_{\gamma}(\gamma)=1-\exp (-a \gamma),
\end{aligned}
$$

where

$$
a=1 / E\left[\gamma_{j}\right]=1 / \bar{\gamma}
$$

To compute the average BS data rate per TTI in the threshold-based multiuser scheduling scheme given by Eq. (4), we first condition on a fixed $n$ and write

$$
\begin{aligned}
r & =E_{n}\left\{E_{\gamma_{1: L}, \gamma_{2: L}, \cdots, \gamma_{n: L}}\left[m \sum_{l=1}^{n} \log _{2}\left(1+\gamma_{l: L}\right) \mid n\right]\right\} \\
& =\sum_{n=1}^{L} \phi(n) \cdot \operatorname{Pr}(\mathbf{n}=n),
\end{aligned}
$$

$$
\phi(n)=E_{\gamma_{1: L}, \gamma_{2: L}, \cdots, \gamma_{n: L}}\left[m \sum_{l=1}^{n} \log _{2}\left(1+\gamma_{l: L}\right) \mid n\right],
$$

and $\operatorname{Pr}(\mathbf{n}=n)$ denotes the probability that there are $n$ users whose SNR equal or exceed $\gamma_{t h}=\mu \gamma_{1: L}$.

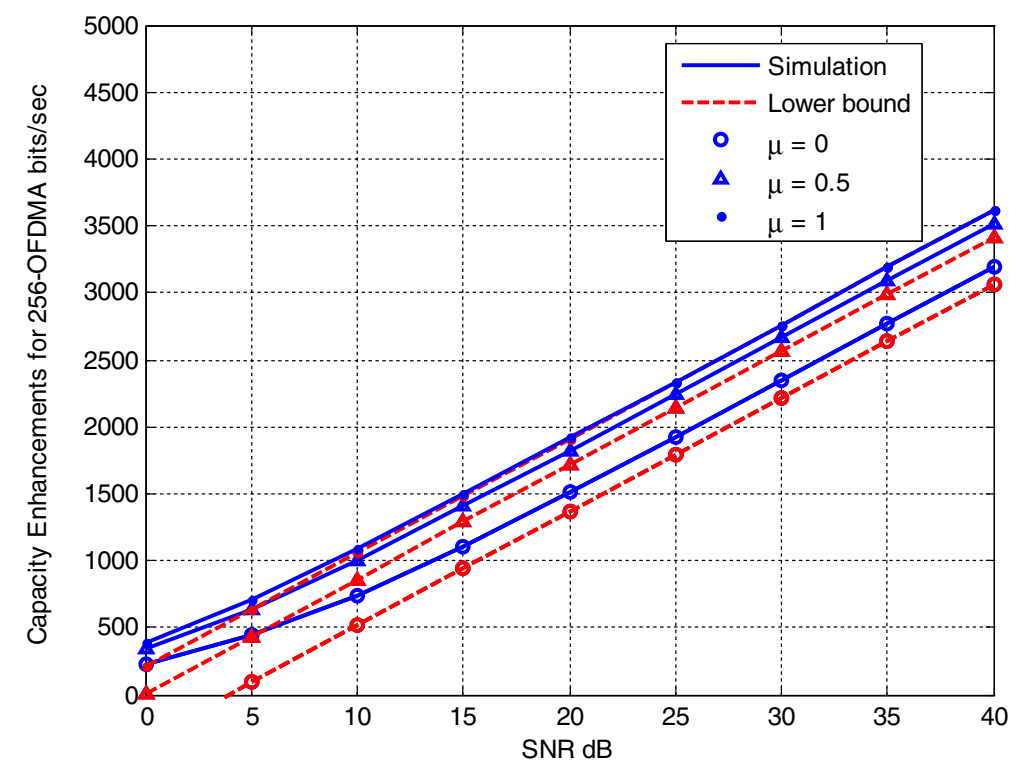

Figure 2 Analytical estimates of the capacity enhancement using threshold-based SNR scheduler in LTE system. 
To compute $\operatorname{Pr}(\mathbf{n}=n)$, we first observe that the event that the random variable $\mathbf{n}=n$ occurs when the following conditions are simultaneously satisfied [7-12]:

$$
\mu \gamma_{1: L} \leq \gamma_{2: L} \leq \gamma_{1: L}, \quad \mu \gamma_{1: L} \leq \gamma_{3: L} \leq \gamma_{2: L}, \cdots, \mu \gamma_{1: L} \leq \gamma_{n: L} \leq \gamma_{n-1: L},
$$

and

$$
0 \leq \gamma_{n+1: L} \leq \mu \gamma_{1: L}, \quad 0 \leq \gamma_{n+2: L} \leq \gamma_{n+1: L}, \quad \ldots, \quad 0 \leq \gamma_{L: L} \leq \gamma_{L-1: L}
$$

Using Eq. (15) and (12), we compute $\operatorname{Pr}(\mathbf{n}=n)$ in [1] as

$$
\begin{aligned}
\operatorname{Pr}(\mathbf{n}=n)= & n\left(\begin{array}{l}
L \\
n
\end{array}\right) \sum_{i=0}^{L-n}(-1)^{i}\left(\begin{array}{l}
L-n \\
i
\end{array}\right) \sum_{j=0}^{n-1}(-1)^{j} \\
& \times\left(\begin{array}{l}
n-1 \\
j
\end{array}\right) \cdot \frac{1}{[1+j+\mu(n-1-j+i)]}
\end{aligned}
$$

Also using Eq. (6), we compute $\phi(n)$ as

$$
\begin{aligned}
\phi(n)= & m \int_{0}^{\infty} \int_{\gamma_{n: L}}^{\infty} \cdots \int_{\gamma_{2: L}}^{\infty} \sum_{l=1}^{n} \log _{2}\left(1+\gamma_{l: L}\right) f_{\gamma_{1: L}, \gamma_{2: L}, \cdots, \gamma_{n: L}} \\
& \left(\gamma_{1: L}, \gamma_{2: L}, \cdots, \gamma_{n: L}\right) d \gamma_{1: L} \cdots d \gamma_{n-1: L} d \gamma_{n: L} \\
= & \int_{0}^{\infty} \int_{\gamma_{n: L}}^{\infty} \cdots \int_{\gamma_{2: L}}^{\infty} \sum_{l=1}^{n} \log _{2}\left(1+\gamma_{l: L}\right) n !\left(\begin{array}{l}
L \\
n
\end{array}\right)\left[1-\exp \left(-a \gamma_{n: L}\right)\right]^{L-n} \\
& \prod_{l=1}^{n} a \exp \left(-a \gamma_{l: L}\right) d \gamma_{1: L} \cdots d \gamma_{n: L}
\end{aligned}
$$

Consider the solution of the integral

$$
\begin{aligned}
I(n)= & \int_{0}^{\infty} \int_{\gamma_{n: L}}^{\infty} \cdots \int_{\gamma_{2: L}}^{\infty} \sum_{l=1}^{n} \log _{2}\left(1+\gamma_{l: L}\right) \\
& n !\left(\begin{array}{c}
L \\
n
\end{array}\right)\left[1-\exp \left(-a \gamma_{n: L}\right)\right]^{L-n} \prod_{l=1}^{n} a \exp \left(-a \gamma_{l: L}\right) d \gamma_{1: L} \cdots d \gamma_{n: L}
\end{aligned}
$$

In Appendix A, we derive an approximate closed-form solution for this integral as

$$
\begin{aligned}
I(n) \geq & \left(\frac{1}{\bar{\gamma}}\right)^{n} n !\left(\begin{array}{l}
L \\
n
\end{array}\right) \frac{1}{\ln 2}\left\{\ln (n !) \prod_{l=1}^{n-1}\left(\frac{\bar{\gamma}}{l}\right) \cdot \bar{\gamma} B[n,(L-n+1)]\right. \\
& +v_{n} \prod_{l=1}^{n-1}\left(\frac{\bar{\gamma}}{l}\right) \cdot \sum_{q=0}^{L-n}\left(\begin{array}{l}
L-n \\
q
\end{array}\right)(-1)^{q}\left(\frac{-\bar{\gamma}}{(q+n)}\left[\mathbf{c}+\ln \left(\frac{1}{\bar{\gamma}}(q+n)\right)\right]\right) \\
& \left.+\sum_{k=1}^{n-1} v_{k} \prod_{l=1, l \neq k}^{n-1}\left(\frac{\bar{\gamma}}{l}\right) \cdot\left(\frac{-\bar{\gamma}}{k}\left[\mathbf{c}+\ln \frac{k}{\bar{\gamma}}\right]\right)(\bar{\gamma} B[n,(L-n+1)])\right\} .
\end{aligned}
$$

Substituting Eq. (16) in Eq. (14), and using Eqs. (10) and (13), we obtain a closed-form expression for the capacity of threshold-based SNR scheduler per TTI in LTE systems as

$$
\begin{aligned}
r \geq & m \sum_{n=1}^{L}\left(( n ) ! ( \frac { 1 } { \overline { \gamma } } ) ^ { n } ( \begin{array} { l } 
{ L } \\
{ n }
\end{array} ) \frac { 1 } { \operatorname { l n } 2 } \left\{\ln (n !) \prod_{l=1}^{n-1}\left(\frac{\bar{\gamma}}{l}\right) \cdot \bar{\gamma} B[n,(L-n+1)]\right.\right. \\
& +v_{n} \prod_{l=1}^{n-1}\left(\frac{\bar{\gamma}}{l}\right) \cdot \sum_{q=0}^{L-n}\left(\begin{array}{l}
L-n \\
q
\end{array}\right)(-1)^{q} \frac{-\bar{\gamma}}{(q+n)}[\mathbf{c}+\ln (1 \bar{\gamma}(q+n))] \\
& \left.\left.+\sum_{k=1}^{n-1} v_{k} \prod_{l=1, l \neq k}^{n-1}\left(\frac{\bar{\gamma}}{l}\right) \cdot\left(\frac{-\bar{\gamma}}{k}[\mathbf{c}+\ln k \bar{\gamma}]\right)(\bar{\gamma} B[n,(L-n+1)])\right\} \cdot \operatorname{Pr}(n)\right),
\end{aligned}
$$

where $\operatorname{Pr}(n)$ is given by Eq. (13). Finally, the total estimate of the BS capacity enhancements over all the TTI's can be estimated for the threshold-based SNR scheduler using Eq. (3).

\section{Simulation results}

\section{Numerical simulations}

In Figure 2, we plot the (lower-bound) analytical results for the capacity of threshold-based SNR scheduler in LTE OFDMA systems, using Eqs. (24) and (13). Counterpart results from numerical simulations of Eq. (4) are also presented in these figures for reference. It is observed from these figures that the lowerbound analytical expressions derived are very close to the actual numerical simulation of the BS capacity with only $5 \%$ gap maximum in high SNR, for the case $\mu=0$ which has the widest gap between the analysis and simulations. All other cases of $\mu$ have even much narrower gaps between the analysis and simulation. The case $\mu=1$ gives exact match between the analysis and simulations in high SNR. Thus the derived expressions are very useful for estimating the capacity enhancements of the proposed threshold-based SNR scheduler at different values of the threshold. As expected, the capacity enhancement increases as the threshold level is increased in the range $0 \leq \mu \leq 1$. The extreme ends $\mu=0$ and $\mu=1$ corresponds to the $R R$ and MaxSNR scheduler as shown in the next figures, while other values of the threshold in this range can be used to model a variety of user fairness vs. capacity enhancements that are not readily modeled in the existing algorithms such as PF.

\section{System-level simulations}

For our system-level simulation, we employed the MATLAB-based LTE PHY Lab and LTE MAC Lab software supplied by IS-wireless [14]. The LTE PHY and MAC Labs are sets of MATLAB-based software implemented to model the whole of the LTE standard, both the physical layer specifications (LTE PHY Lab) and the Medium Access layer specifications (LTE MAC Lab). The details of the parameters used in our simulations are as shown in Table 1. Figure 3 presents the 
Table 1 System level simulation parameters for threshold-based scheduling in LTE

\begin{tabular}{ll}
\hline System level simulation parameters & \\
\hline Transmission frequency & $850 \mathrm{MHz}$ \\
FFT size & 256 \\
No. of cells in cluster & 7 (6 interfering BS's) \\
Transmission Bandwidth & $3 \mathrm{MHz}$ \\
Duplexing & FDD \\
Environment & Urban \\
Propagation model & $3 \mathrm{GPP}$ model \\
Multipath model & $3 \mathrm{GPP}$ model \\
BS antenna Characteristics & Omni directional \\
Height of BS & $30 \mathrm{~m}$ \\
Transmission power of BS (dBm) & 46 \\
Height of UE's & $2 \mathrm{~m}$ \\
Transmission power of UE's (dBm) & 23 \\
Speed of UE's (Km/h) & UE $1=$ stationary \\
& UE $2=45 \mathrm{~km} / \mathrm{h}$ \\
& UE $3=100 \mathrm{~km} / \mathrm{h}$ \\
\hline
\end{tabular}

simulation results obtained for the BS sum rate throughput, over nine TTI's considered in our simulation, defined as:

$$
C_{B S}=\sum_{t=1}^{N_{T T I}} \sum_{k=1}^{N_{c}} \log _{2}\left(1+S I N R_{k}\right)
$$

where SINR denotes the signal-to-interference plus noise ratio. In the simulation, we were able to incorporate the effects interference from neighboring BS, which could not be done in the analysis due to analytical difficulties. However, as shown in this figure, it can be observed that in general, the trends observed in the analysis are also confirmed by the system-level simulation results. As a reference, we have also included the performance of popular LTE scheduling schemes such as the RR, MaxSNR, and the PF schemes in this figure. It should be mentioned that the results presented here for RR, MaxSNR, and PF schemes were obtained directly from the LTE simulator used, which already has these algorithms implemented on its list of existing LTE schedulers. Thus we do not need to model those algorithms in our simulation but to focus on the proposed threshold-based SNR scheduler. As confirmed in the results in Figure 3, MaxSNR and RR have the same performance as the proposed threshold-based SNR scheduling scheme for $\mu=1$ and $\mu=1$ respectively, while various levels of PF can be modeled for different values of $\mu$.

In Table 2, we display the BS sum rate throughput achieved over 9 TTI's by different LTE schedulers, in bits per seconds per Hertz (bps/Hz). The trends depicted in Figure 3 are also confirmed in this table. Notice also from the results in this table that Eq. (3) provides a good approximation for the overall BS capacity for all values of the threshold, $\mu$.

In Figures 4, 5, 6, 7, we compare the number of assigned PRB's over nine TTI's in the threshold-based SNR scheduler with existing LTE schedulers. It should be noted from our simulation set-up in Table 1 that user 1 (denoted as UE 1 in Figures 4, 5, 6, 7) has the best simulated channel, followed by user 2 (UE 2), and then

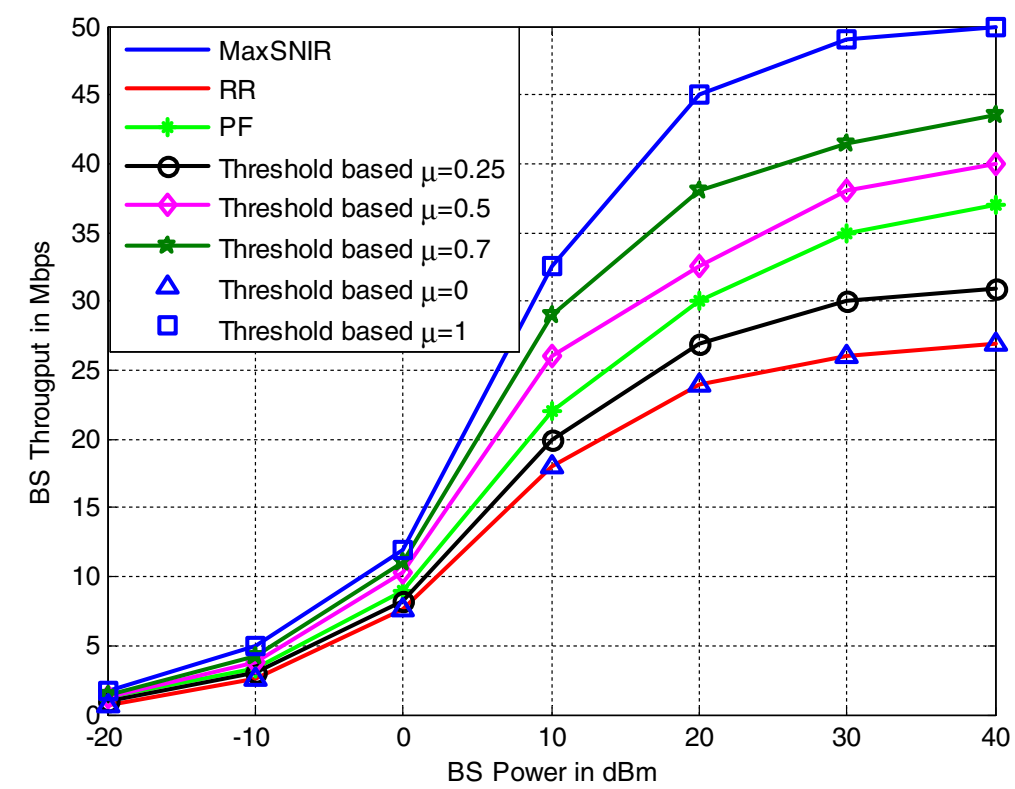

Figure 3 System-level simulation results for threshold-based SNR scheduling in LTE system. 
Table 2 BS sum rate throughput achieved over 9 TTI's by different LTE schedulers

\begin{tabular}{lcc}
\hline Scheduler type & Overall BS throughput & BS throughput per TTI (randomly selected) \\
\hline MaxSNIR & $154 \mathrm{bps} / \mathrm{Hz}$ & $18 \mathrm{bps} / \mathrm{Hz}$ \\
Round Robin & $78 \mathrm{bps} / \mathrm{Hz}$ & $9 \mathrm{bps} / \mathrm{Hz}$ \\
Proportional Fairness & $118 \mathrm{bps} / \mathrm{Hz}$ & $14 \mathrm{bps} / \mathrm{Hz}$ \\
Threshold-based SNR with $\mu=1$ & $154 \mathrm{bps} / \mathrm{Hz}$ & $18 \mathrm{bps} / \mathrm{Hz}$ \\
Threshold-based SNR with $\mu=0.9$ & $149 \mathrm{bps} / \mathrm{Hz}$ & $17 \mathrm{bps} / \mathrm{Hz}$ \\
Threshold-based SNR with $\mu=0.8$ & $144 \mathrm{bps} / \mathrm{Hz}$ & $16 \mathrm{bps} / \mathrm{Hz}$ \\
Threshold-based SNR with $\mu=0.7$ & $135 \mathrm{bps} / \mathrm{Hz}$ & $15 \mathrm{bps} / \mathrm{Hz}$ \\
Threshold-based SNR with $\mu=0.6$ & $129 \mathrm{bps} / \mathrm{Hz}$ & $15 \mathrm{bps} / \mathrm{Hz}$ \\
Threshold-based SNR with $\mu=0.5$ & $124 \mathrm{bps} / \mathrm{Hz}$ & $14 \mathrm{bps} / \mathrm{Hz}$ \\
Threshold-based SNR with $\mu=0.4$ & $111 \mathrm{bps} / \mathrm{Hz}$ & $13 \mathrm{bps} / \mathrm{Hz}$ \\
Threshold-based SNR with $\mu=0.3$ & $103 \mathrm{bps} / \mathrm{Hz}$ & $12 \mathrm{bps} / \mathrm{Hz}$ \\
Threshold-based SNR with $\mu=0.2$ & $93 \mathrm{bps} / \mathrm{Hz}$ & $11 \mathrm{bps} / \mathrm{Hz}$ \\
Threshold-based SNR with $\mu=0.1$ & $84 \mathrm{bps} / \mathrm{Hz}$ & $10 \mathrm{bps} / \mathrm{Hz}$ \\
Threshold-based SNR with $\mu=0$ & $78 \mathrm{bps} / \mathrm{Hz}$ & $9 \mathrm{bps} / \mathrm{Hz}$
\end{tabular}

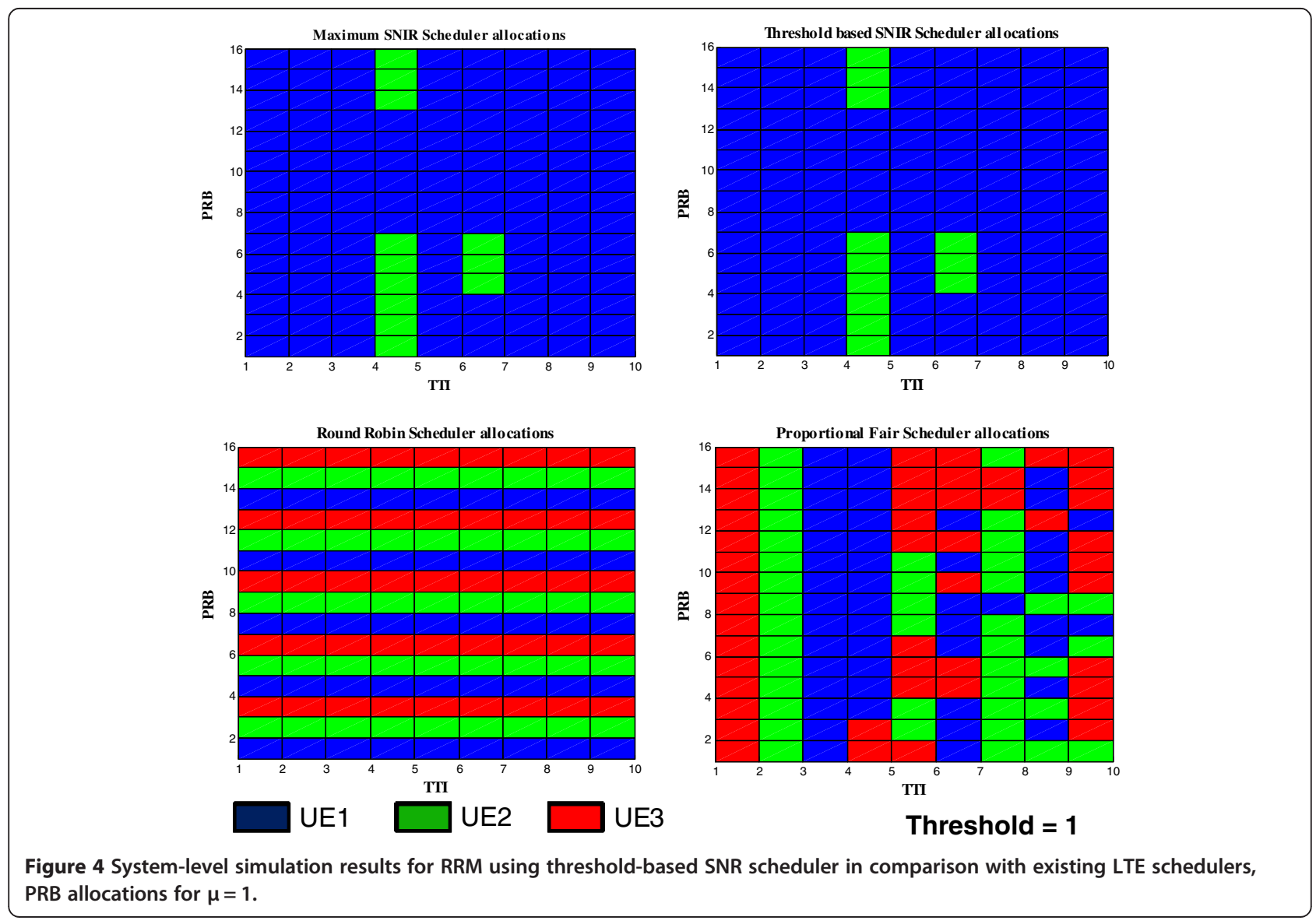



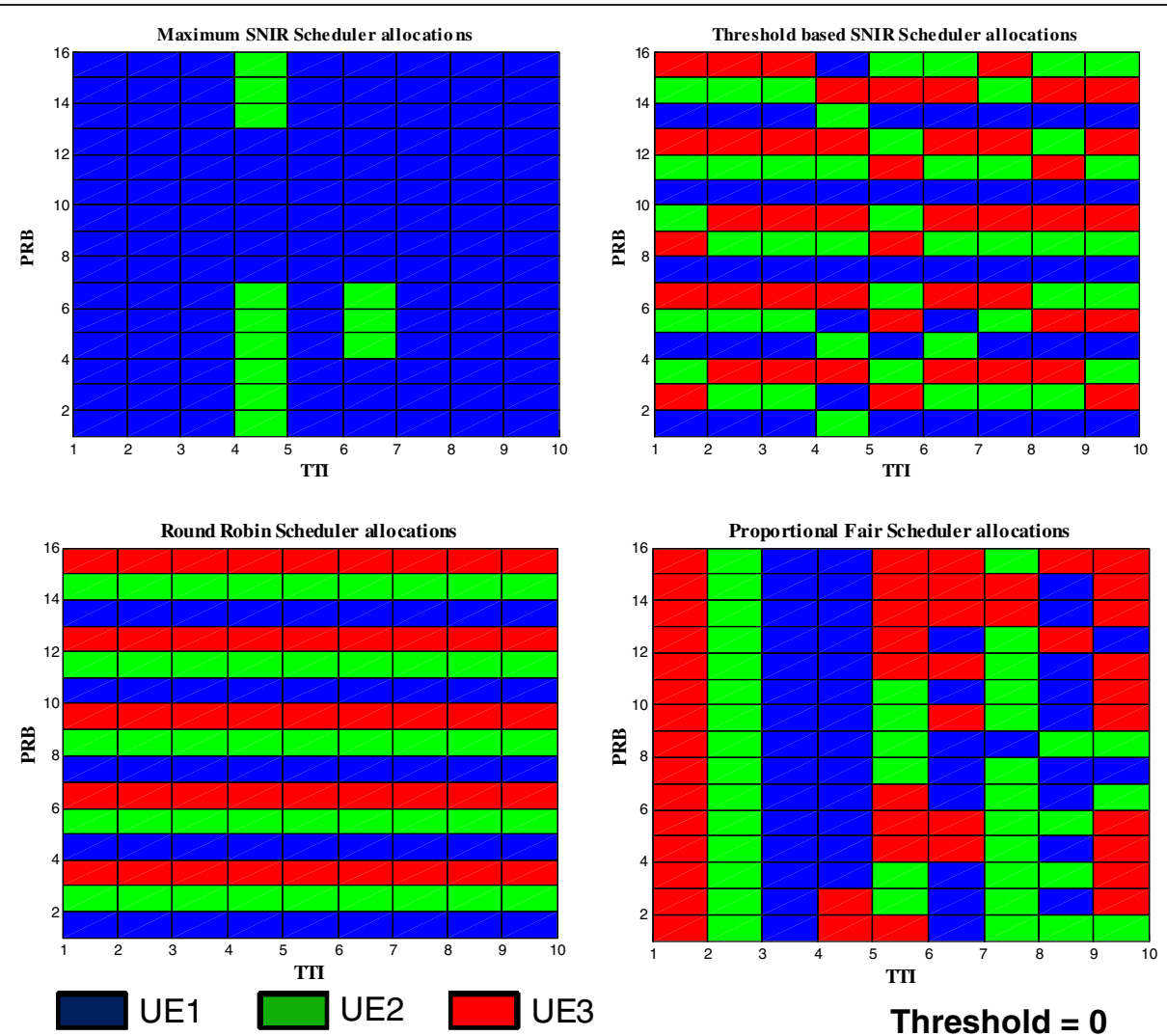

Figure 5 System-level simulation results for RRM using threshold-based SNR scheduler in comparison with existing LTE schedulers, PRB allocations for $\mu=0$.

user 3 (UE 3). Thus, scheduling algorithms that tend to assign more PRBs to UE 1 are in effect maximizing BS data rate at the expense of user fairness, while those that tend to assign the PRBs evenly across all the users are in effect maximizing user fairness at the expense of BS data rate enhancements. In the simulation experiments in Figures $4,5,6,7$, we compare the behaviors of the proposed threshold-based SNR scheduler with other LTE schedulers, while operating over these two extreme ends.

From Figure 4, it is observed that for $\mu=1$, the number of assigned PRB's for each user using the thresholdbased scheduler and the MaxSNR scheduler are exactly the same in sequence of assignments and in quantity $(\mathrm{UE} 1=123$, UE2 $=12$, UE3 $=0$ ), as expected. This again confirms the equivalence between the MaxSNR scheduler and the threshold-based SNR scheduler for $\mu=1$.

From Figure 5, it is observed that for $\mu=1$, the number of assigned PRB's for each user using the threshold-based SNR scheduler and the RR scheduler are exactly the same in quantity (UE1 $=45, \mathrm{UE} 2=45$, $\mathrm{UE} 3=45$ ), even though they are not necessarily the same in sequence because of the random variations in the user channel statistics over the different PRB's. Again, this also confirms the equivalence between the RR scheduler and the threshold-based SNR scheduler for $\mu=1$.

\section{Fairness issues}

As $\mu$ assumes different values in between the two extreme ends $(0<\mu<1)$, different flavor of user fairness vs. capacity enhancements not captured by the existing PF scheme, can be modeled by the proposed thresholdbased SNR scheduler. For example in Figure 6, it is observed that threshold-based SNR scheduler for $\mu=0.2$ achieves a good measure of user fairness for all users while at the same time enhancing BS capacity significantly more than the PF scheduler, with the following PRB assignments: UE $1=73$, UE $2=34, \mathrm{UE} 3=28$. The respective $P R B$ assignments for each user in the existing PF are: UE $1=48$, UE2 $=40$, UE3 $=47$, which is just a bit fairer but with much less BS capacity enhancements. Similarly in Figure 7, threshold-based SNR scheduler for $\mu=0.5$ achieves a bit less user fairness 
but with much more capacity enhancements than the PF scheduler.

\section{Implementation issues}

Notice that MaxSNR, threshold-based SNR, and PF, all require the availability of user channel state information at the BS, and this is all that is required for implementing a threshold-based SNR scheduler at the BS. Thus implementation-wise, threshold-based SNR scheduler does not require any additional complexity compared to the existing schedulers, despite its ability to model several other scheduling scenarios. On the contrary, as explained in the system model and analysis section, since a scheduling decision is good across $n$ adjacent PRBs in frequency domain, scheduling decision computations can be made faster in the thresholdbased SNR scheduler than the existing schedulers that compute scheduling decisions for each and every PRBs. This advantage becomes more noticeable in OFDMA systems with large FFT size such as 1024-OFDMA or 2048-ODMA. Therefore, it is hoped that a thresholdbased scheduler when implemented at the BS would provide a simpler, yet efficient, generalized scheduling policy that would combine the separate implementations of MaxSNR, PF, and RR, currently available in LTE BS $[15,16]$, into one simple algorithm where the threshold definition simply dictates the specific scheduling realization such as MaxSNR, RR, PF, etc., to be used at any time instant.

\section{User mobility issues}

In Table 3, we tabulate the effects of user mobility on the behavior of the threshold-based scheduler, after simulating several results similar to Figures 4, 5, 6, 7, but for different values of the threshold. It can be observed from this table that when the scheduler operates with large threshold, such as $\mu>0.5$, the algorithm will seek high capacity enhancement (or less user fairness), and thus users with high mobility tend to be given fewer number of PRB's. On the other hand when the scheduler operates with small threshold, such as $\mu \leq 0.5$, the algorithm will seek low capacity enhancement (or high user fairness), and thus users with high mobility tend to be given similar number of PRB's as those with low mobility. The result in this table, again emphasize the wide range of user fairness vs. capacity enhancements possible using the threshold-based SNR scheduler.
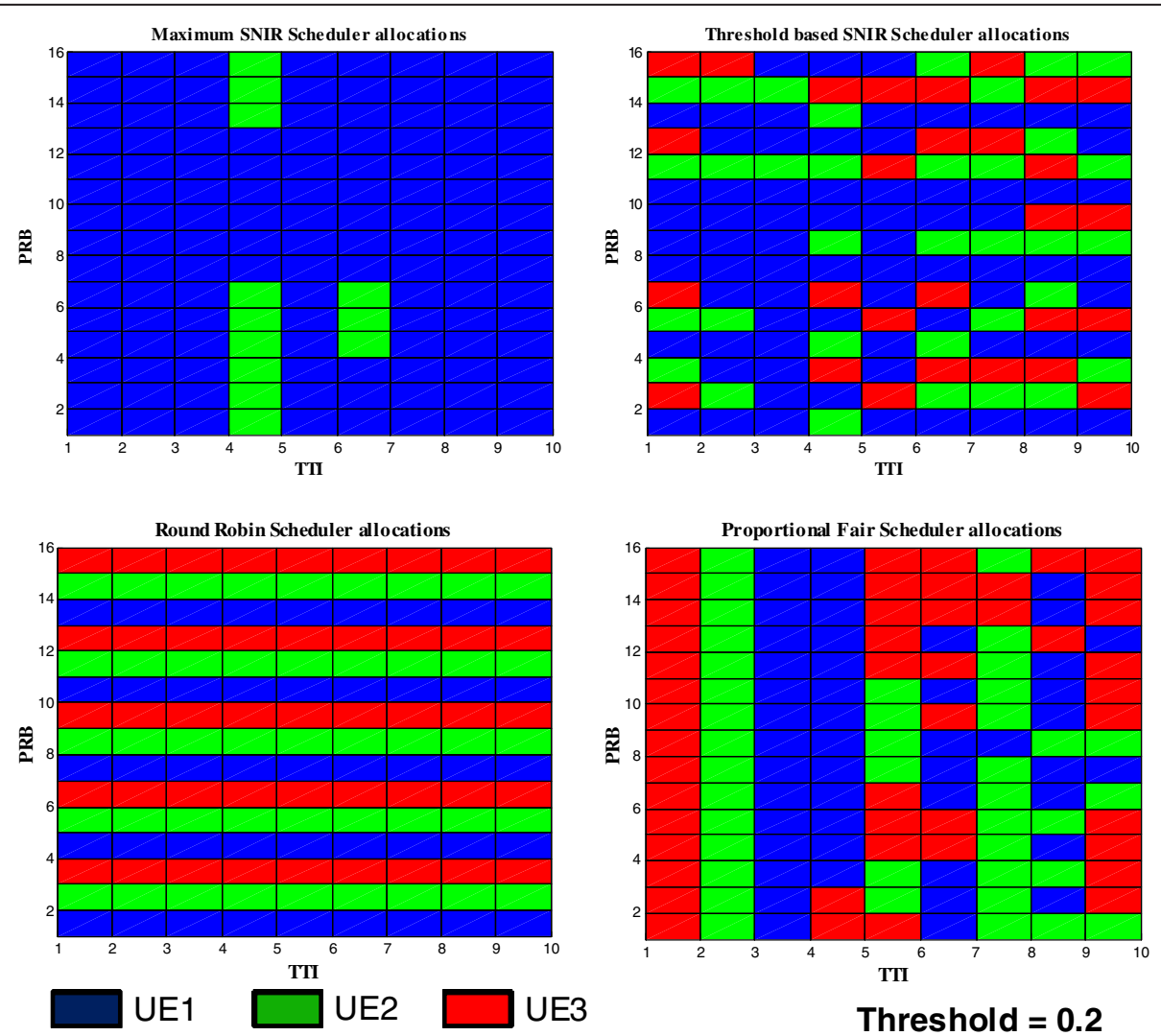

Figure 6 System-level simulation results for RRM using threshold-based SNR scheduler in comparison with existing LTE schedulers, PRB allocations for $\mu=0.2$. 

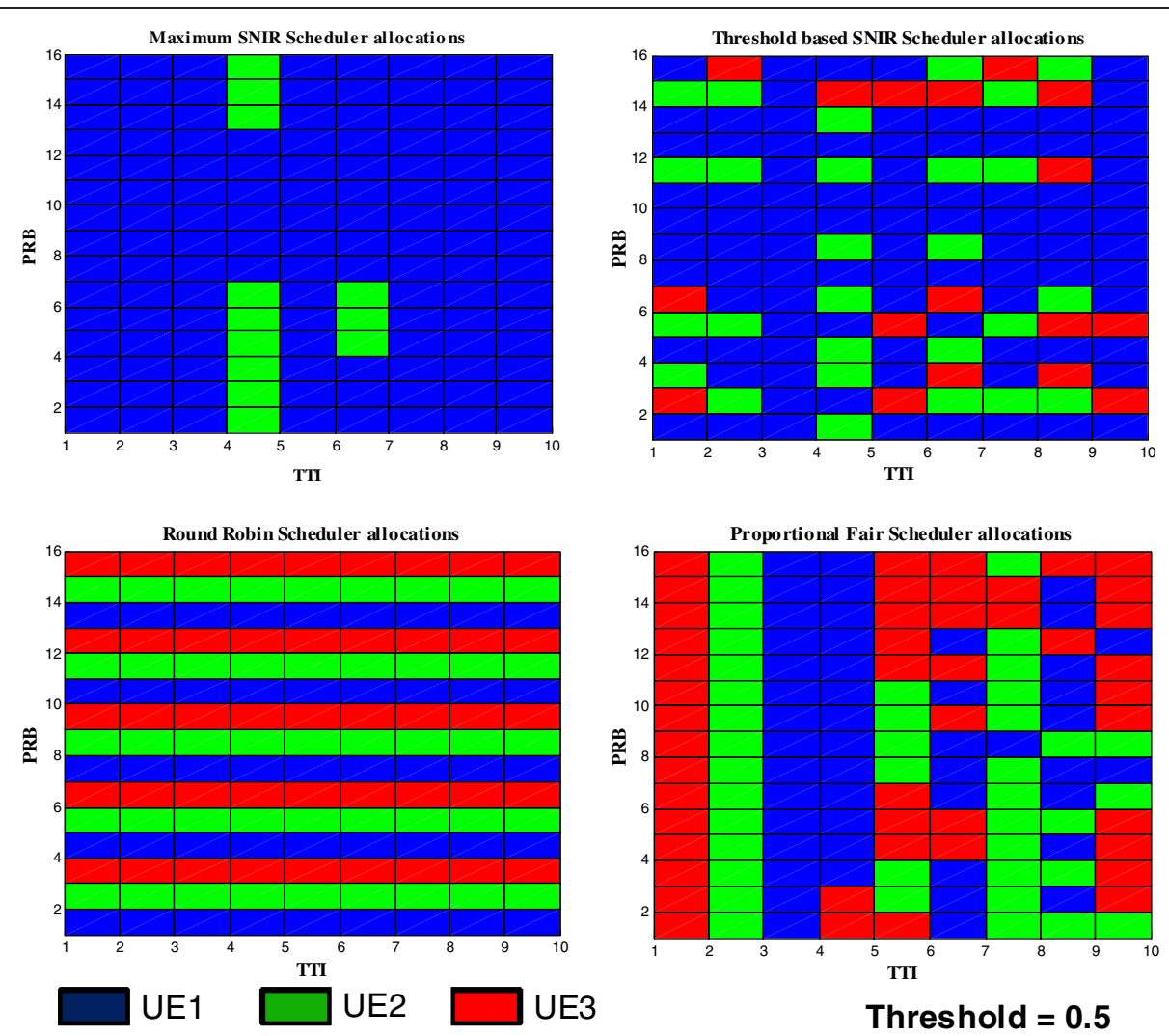

Figure 7 System-level simulation results for RRM using threshold-based SNR scheduler in comparison with existing LTE schedulers, PRB allocations for $\mu=0.5$.

\section{Conclusions}

This paper proposes a threshold-based SNR scheduling scheme for downlink transmission in LTE OFDMA systems. In the proposed threshold-based SNR scheduling scheme, the BS scheduler selects at any time instant users whose channel gains in the available PRBs equal or exceed a pre-determined energy threshold. The threshold definition can be used to maximize BS capacity on the available PRBs, or to enhance user fairness. We quantify analytically the capacity enhancements provided by the proposed scheme for different SNR threshold levels, and also present numerical simulation results to verify the analysis. Furthermore, we present system-level simulations that accurately model all the LTE physical and MAC layer parameters stipulated in the standard, to test the behaviour of the proposed scheduling scheme

Table 3 Effect of User (UE) mobility on threshold-based SNR scheduling in LTE

\begin{tabular}{lccc}
\hline Threshold & $\begin{array}{c}\text { PRB assigned UE1 } \\
\text { (speed =0 } \mathbf{~ k m} / \mathbf{h})\end{array}$ & $\begin{array}{c}\text { PRB assigned UE2 } \\
\text { (speed = 45 } \mathbf{~ k m} / \mathbf{h})\end{array}$ & $\begin{array}{r}\text { PRB assigned UE3 } \\
\text { (speed = 100 } \mathbf{~ k m} / \mathbf{h})\end{array}$ \\
\hline Threshold-based SNR with $\mu=1$ & 123 & 12 & 0 \\
Threshold-based SNR with $\mu=0.9$ & 116 & 16 & 3 \\
Threshold-based SNR with $\mu=0.7$ & 103 & 21 & 11 \\
Threshold-based SNR with $\mu=0.5$ & 91 & 27 & 17 \\
Threshold-based SNR with $\mu=0.4$ & 86 & 28 & 21 \\
Threshold-based SNR with $\mu=0.2$ & 73 & 34 & 28 \\
Threshold-based SNR with $\mu=0$ & 45 & 45 & 45 \\
\hline
\end{tabular}


when employed in LTE networks. Both the analyses and simulations indicate that a threshold-based SNR scheduler when implemented at the BS would provide a simpler, yet efficient, generalized scheduling policy that could combine the separate implementations of the existing LTE schedulers such as the MaxSNR, RR, and $\mathrm{PF}$, into one single scheduling algorithm where the threshold definition simply dictates the specific scheduling policy such as MaxSNR, RR, PF, etc., to be realized at any time instant.

\section{Appendix A}

To solve Eq. (15), we consider the transformation of the random variables $\left\{\gamma_{l: L}\right\}_{l=1}{ }^{n}$ obtained by defining the spacings [17]

$$
\begin{aligned}
Y_{1} & =\gamma_{1: L}-\gamma_{2: L}, \quad Y_{2}=\gamma_{2: L}-\gamma_{3: L}, \quad \ldots, \quad Y_{n-1} \\
& =\gamma_{n-1: L}-\gamma_{n: L}, \quad Y_{n}=\gamma_{n: L} .
\end{aligned}
$$

It can be shown that the random variables $Y_{1}, Y_{2}, \cdots Y_{n}$ are all statistically independent, with $p d f$ given by [17]

$$
f_{Y_{l}}\left(y_{l}\right)=a l \exp \left(-a l y_{l}\right)
$$

where $y_{l} \geq 0, l=1, \cdots, n$.Using Eqs. (19) and (20), Eq. (15) can be expressed as

$$
\begin{aligned}
I(n)= & \int_{0}^{\infty} \cdots \int_{0}^{\infty}\left[\log _{2}\left(1+y_{1}+\cdots+y_{n}\right)+\log _{2}\right. \\
& \left.\times\left(1+y_{2}+\cdots+y_{n}\right)+\cdots+\log _{2}\left(1+y_{n-1}+y_{n}\right)+\log _{2}\left(1+y_{n}\right)\right] \\
\times & n !\left(\begin{array}{l}
L \\
n
\end{array}\right)\left[1-\exp \left(-a y_{n}\right)\right]^{L-n} \prod_{l=1}^{n} \exp \left(- \text { aly }_{l}\right) \text { day }_{1} \cdots \text { day }_{n} \\
\approx & \int_{0}^{\infty} \cdots \int_{0}^{\infty}\left[\log _{2}\left\{\left(y_{1}+\cdots+y_{n}\right) \cdot\left(y_{2}+\cdots+y_{n}\right) \cdots \cdot\left(y_{n-1}+y_{n}\right) \cdot\left(y_{n}\right)\right\}\right] \\
& \times n !\left(\begin{array}{l}
L \\
n
\end{array}\right)\left[1-\exp \left(-a y_{n}\right)\right]^{L-n} \prod_{l=1}^{n} \exp \left(- \text { aly }_{l}\right) \text { day }_{1} \cdots \text { day }_{n} .
\end{aligned}
$$

The product of partial sum in Eq (21) is characterized in $[19,20]$, but the extra term $\left[1-\exp \left(-a y_{n}\right)\right]^{L-n}$ in Eq. (21) makes the result not exactly applicable here. However, using the arithmetic-geometric mean inequality (with equality when $y_{1}=y_{2}=y_{n}$, i.e. fully correlated user channels), a guaranteed lower bound on $I(n)$ can be obtained as [18] which can be written as

$$
\begin{aligned}
I(n) & \geq \int_{0}^{\infty} \cdots \int_{0}^{\infty} \frac{1}{\ln 2}\left[\ln (n !)+\ln \left\{\left(y_{n}\right)^{v_{n}} \cdot\left(y_{n-1}\right)^{v_{n-1}} \cdots \cdot\left(y_{2}\right)^{v_{2}} \cdot\left(y_{1}\right)^{v}\right\}\right] \\
& \times(a)^{n} n !\left(\begin{array}{l}
L \\
n
\end{array}\right)\left[1-\exp \left(-a y_{n}\right)\right]^{L-n} \exp \left[-a\left(y_{1}+2 y_{2}+\cdots+n y_{n}\right)\right] d y_{1} \cdots d y_{n} \\
& =\int_{0}^{\infty} \cdots \int_{0}^{\infty} \frac{1}{\ln 2}\left[\ln (n !)+\sum_{l=1}^{n} v_{l} \ln \left(y_{l}\right)\right] \\
& \times(a)^{n} n !\left(\begin{array}{l}
L \\
n
\end{array}\right)\left[1-\exp \left(-a y_{n}\right)\right]^{L-n} \exp \left[-a\left(y_{1}+2 y_{2}+\cdots+n y_{n}\right)\right] d y_{1} \cdots d y_{n}
\end{aligned}
$$

where $v_{n}=\sum_{k=1}^{n} \frac{1}{k}, \quad v_{n-1}=\sum_{k=2}^{n} \frac{1}{k}, \ldots, \quad v_{2}=\sum_{k=n-1}^{n} \frac{1}{k}$, $v_{1}=\sum_{k=n}^{n} \frac{1}{k}$.

Thus the integrals in Eq. (22), can be partitioned as

$$
\begin{aligned}
I(n) \geq(a)^{n} n !\left(\begin{array}{l}
L \\
n
\end{array}\right) \frac{1}{\ln 2}\left\{\ln (n !) \int_{0}^{\infty} \cdots \int_{0}^{\infty}\left[1-\exp \left(-a y_{n}\right)\right]^{L-n}\right. \\
+v_{n} \int_{0}^{\infty} \cdots \int_{0}^{\infty} \ln y_{n}\left[1-\exp \left(-a y_{n}\right)\right]^{L-n} \\
\left.+\sum_{k=1}^{n-1} v_{k} \int_{0}^{\infty} \cdots \int_{0}^{\infty} \ln y_{k}\left[1-\exp \left(-a y_{n}\right)\right]^{L-n}\right\} \\
\left.=(a)^{n} n !\left(y_{n}+2 y_{2}+\cdots+n y_{n}\right)\right] d y_{n} \frac{1}{\ln 2}\left\{\ln (n !) \prod_{l=1}^{n-1}\left(\frac{1}{a l}\right) \cdot \int_{0}^{\infty}\left[1-\exp \left(-a y_{n}\right)\right]^{L-n}\right. \\
+v_{n} \prod_{l=1}^{n-1}\left(\frac{1}{a l}\right) \cdot \int_{0}^{\infty} \ln y_{n}\left[1-\exp \left(-a y_{n}\right)\right]^{L-n} \exp \left[-a n y_{n}\right] d y_{n} \\
+\sum_{k=1}^{n-1} v_{k} \prod_{l=1, l \neq k}^{n-1}\left(\frac{1}{a l}\right) \cdot\left(\int_{0}^{\infty} \ln y_{k} \exp \left[-a k y_{k}\right] d y_{k}\right) \\
\left.\quad \times\left(\int_{0}^{\infty}\left[1-\exp \left(-a y_{n}\right)\right]^{L-n} \exp \left[-a n y_{n}\right] d y_{n}\right)\right\} .
\end{aligned}
$$

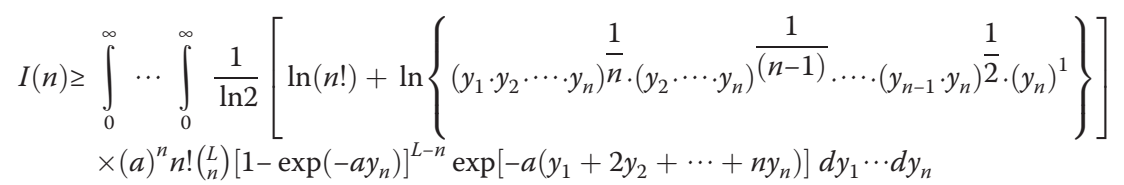


Substituting the following results from [21], Eq. 3.312-1, 4.331-1 into Eq. (24),

$$
\begin{aligned}
I_{l} & =\int_{0}^{\infty} \ln y_{l} \exp \left[-a l y_{l}\right] d y_{l}=\frac{-1}{a l}[\mathbf{c}+\ln a l] ; \quad \mathbf{c} \approx 0.5772 . \\
I_{L n} & =\int_{0}^{\infty}\left[1-\exp \left(-a y_{n}\right)\right]^{L-n} \exp \left[-a n y_{n}\right] d y_{n} \\
& =\frac{1}{a} B[n,(L-n+1)] ; \quad B=\int_{0}^{1} t^{x-1}(1-t)^{y-1} d t,
\end{aligned}
$$

we arrive at the final closed-form results in Eq. (16) for $I(n)$, after some algebra.

\section{Competing interests}

The authors declare that they have no competing interests.

\section{Acknowledgments}

This work is sponsored by grant from the National Plan for Science and Technology (NPST), King Saud University, grant no. 09-ELE302.

\section{Author details}

'Department of Electrical Engineering, King Saud University, Riyadh, Saudi Arabia. ${ }^{2}$ KACST Technology Innovation Center RFTONICS, King Saud University, Riyadh, Saudi Arabia. ${ }^{3}$ School of Computing, Queen's University at Kingston, Ontario, Canada.

\section{Received: 6 September 2012 Accepted: 6 March 2013}

Published: 22 April 2013

\section{References}

1. Al Sulyman, "Throughput Gain using Threshold-based Multiuser Scheduling in WiMAX OFDMA,". EURASIP J. Wirel. Commun. Netw. 2011, 941350 (2011)

2. M Guizan, M Hamdi, P Lorenz, M Ma, Wireless brodband access: WiMAX and beyond. IEEE Commun. Mag. 45(12), 60-61 (2007)

3. M Salah, NA Ali, A-E Taha, H Hassanein, Evaluating Uplink Schedulers in LTE in Mixed Traffic Environments (IEEE-ICC2011, Kyoto, Japan, 2011), pp. 1-5

4. T Bejaoui, A Masmoudi, N Nasser, Radio Resource Optimization and Scheduling Techniques for HSPA and LTE Advanced Technologies, in Evolved Cellular Network Planning and Optimisation for UMTS and LTE, ed. by L Song, J Shen (CRC Press, 2010)

5. Al Sulyman, M Kousa, "Bit Error rate performance of a generalized selection diversity combining scheme in Nakagami fading channels,". Proc., IEEEWCNC, Chicago, USA 3, 1080-1085 (2000)

6. Al Sulyman, M Kousa, Bit Error rate performance analysis of a thresholdbased generalized selection combining scheme in Nakagami fading channels. Eurasip J Wireless Commun Networking 2, 242-248 (2005)

7. MK Simon, M-S Alouini, Performance analysis of generalized selection combining with threshold test per branch (TGSC). IEEE Trans. Veh. Technol. 51(5), 1018-1029 (2002)

8. A Annamalai, G Deora, C Tellambura, "Unified analysis of generalized selection diversity with normalized threshold test per branch". Proc. IEEE WCNC, New Orleans, USA 2, 752-756 (2003)

9. X Zhang, NC Beaulieu, SER of threshold-based hybrid selection/maximalratio combining in equicorrelated Nakagami fading. IEEE Commun. Lett. 8 (9), 552-554 (2004)

10. X Zhang, NC Beaulieu, SER and outage of threshold-based selection/ maximal-ratio combining over generalized fading channels. IEEE Trans. Commun. 52(12), 2143-2153 (2004)

11. X Zhang, NC Beaulieu, SER of threshold-based hybrid selection/maximalratio combining in correlated Nakagami fading. IEEE Trans. Commun. 53(9), 1423-1426 (2005)
12. X Zhang, NC Beaulieu, Performance analysis of generalized selection combining in generalized correlated nakagami-m fading. IEEE Trans. Commun. 54(11), 2103-2111 (2006)

13. L Cao, NC Beaulieu, "Effects of channel estimation errors on absolute threshold-generalized selection diversity combining". ICC, Seoul, Korea 2005(4), 2200-2206 (2005)

14. IS-wireless, LTE PHY Lab ${ }^{T M}$, and "ITE MAC Lab ${ }^{T M}$ (IS-wireless Inc, Warsaw, Poland, 2011)

15. J Wannstrom, "LTE-Advanced," Third Generation Partnership Project (3GPP). website, http://www.3gpp.org/LTE-Advanced, accessed July 2012

16. M Sauter, From GSM to LTE: An Introduction to Mobile Networks and Mobile Broadband (John Wiley and Sons, Ltd, United Kingdom, 2011)

17. N Balakrishnan, AC Cohen, Order Statistics and Inference (Academic Press, Inc, 1991)

18. A Papoullis, SU Pillai, Probability, Random Variables and Stochastic Processes, 4th edn. (Mc Graw-Hill, Inc, 2002)

19. BC Arnold, JA Villasenor, The asymptotic distribution of sums of records. Extremes 1(3), 351-363 (1998)

20. Y Qi, Limit distributions for products of sums. Elsevier Stat Prob Lett 62, 93-100 (2003)

21. IS Gradshteyn, IM Ryshik, Table of Integrals, Series, and Products, 7th edn. (Academia Press, Elsevier Inc, London-UK, 2007)

doi:10.1186/1687-6180-2013-85

Cite this article as: Sulyman et al:: Capacity analysis of threshold-based SNR scheduler in LTE systems. EURASIP Journal on Advances in Signal Processing 2013 2013:85.

\section{Submit your manuscript to a SpringerOpen ${ }^{\odot}$ journal and benefit from:}

- Convenient online submission

- Rigorous peer review

- Immediate publication on acceptance

- Open access: articles freely available online

- High visibility within the field

- Retaining the copyright to your article

Submit your next manuscript at $\gg$ springeropen.com 